\title{
Variations
}

Variations

Revue internationale de théorie critique

11 | 2008

La beauté est dans la rue

\section{Mexique : constellation et lutte de classes, les espoirs du passé au présent, 1968-2008}

Fernando Matamoros

\section{OpenEdition}

Journals

Édition électronique

URL : http://journals.openedition.org/variations/266

DOI : 10.4000/variations.266

ISSN : 1968-3960

Éditeur

Les amis de Variations

Référence électronique

Fernando Matamoros, « Mexique : constellation et lutte de classes, les espoirs du passé au présent 1968-2008», Variations [En ligne], 11 | 2008, mis en ligne le 01 février 2012, consulté le 21 avril 2019. URL : http://journals.openedition.org/variations/266 ; DOI : 10.4000/variations.266

Ce document a été généré automatiquement le 21 avril 2019

Les ami•e•s de Variations 


\title{
Mexique : constellation et lutte de classes, les espoirs du passé au présent, 1968-2008
}

\author{
Fernando Matamoros
}

\section{NOTE DE L'ÉDITEUR}

Première publication sur www.theoriecritique.com, « La beauté est dans la rue », 1er mai 2008, pp. 82-90

\section{NOTE DE L'AUTEUR}

La première version de ce texte a été rédigée à titre de chercheur au département de science politique du Massachusetts Institute of Technology (MIT) et de boursier du Conseil de recherches en sciences humaines du Canada (CRSH).

Le regard de l'ange de l'histoire de Walter Benjamin (Sur le concept d'histoire, Thèse IX) ${ }^{1}$ nous rappelle que l'action révolutionnaire peut arrêter le temps de l'horloge du Capital. L'ange lutte contre le vent du Progrès qui, tout en le poussant, souffle, malgré tout, de nouvelles promesses pour la rédemption des histoires et des morts du passé. L'ange annonce la révolution, une nécessité inscrite dans l'histoire des vaincus, millions de particules à la recherche d'un mieux être. Son regard épouvanté interpelle le désir renouvelé par la douleur des morts, ces blessures du travail-concret, lutte de classes active dans le présent du monde des résistances, délires en ces temps de fantasmagorie totalitaire de la marchandise. Il concrétise les rêves éloignés des conditions objectives de la privatisation et de la domination. Ses yeux, ouverts sur le passé, sont les portes de l'âme par où peut venir la révolution, celle qui n'a pas culminé et dont les pensées rebelles et insoumises et les pas de résistance continuent à demander et chercher comment marcher pour 
atteindre et faire un autre monde de notre monde. Leurs réflexions deviennent actions pour l'instant du pas-encore (Bloch, 1976) au présent.

2 La date de 1968 avec de celle de 2008 peut être un autre rendez-vous du passé avec le présent, un aller au -delà du temps des vainqueurs car il s'agit des recherches d'espoir de l'homme inachevé. Ce sont les images dialectiques en lutte contre les dieux de la marchandise, horrible mythe de la barbarie du capitalisme inhumain.

4 Le massacre du 2 octobre 1968 à Mexico fait partie de ces plaies profondes qui ne cicatrisent pas. Ces blessures, qui ne se sont pas fermées à cette date douloureuse, saignent encore. Elles font partie des pratiques du pouvoir, élément essentiel du rouage du capitalisme. Quarante ans après la révolte de 1968, nous constatons que les morts, images dialectiques du pouvoir du capital, se retournent encore dans leurs tombes, préoccupés et inquiets pour la présence de leur mémoire. En effet, la structure du pouvoir cherche à les enterrer d'avantage et à les enfermer dans le passé statique des shows télévisés des dossiers de l'histoire aseptisés.

5 Les morts et disparus (dont les restes de centaines d'entre eux ne sont toujours pas localisés) sortent de leurs sépulcres pour nous rappeler que l'ennemi n'a pas cessé de vaincre. Ils se demandent encore pourquoi les crimes de ces dates restent impunis, pourquoi la justice reste-t-elle un mot vidé des contenus des luttes, en l'absence des sujets exigeant un châtiment des responsables institutionnels du capital vivant encore protégés par la loi que souffle le vent de la violence de l'époque.

6 Dans ce sens, il n'est ni surprenant ni un hasard que le spectre de la lutte de classes inquiète les chiens de garde de l'ordre établi. Une guerre existe pour l'appropriation des images de l'histoire : une mobilisation de la mémoire contre l'oubli. En France, les « gens respectables » considèrent le symbole de 68 comme le frein du progrès du néolibéralisme ${ }^{2}$ . Le président français, Nicolas Sarkozy, n'a pas hésité à remettre en question ce moment de l'histoire en évoquant le spectre de 1968 comme le responsable de l'« effondrement » de la France en tant que puissance économique. Pour lui, il faut "savoir si l'héritage de Mai 68 doit être perpétué ou s'il doit être liquidé une bonne fois pour toutes " puisqu'il empêche l'accumulation désirée par les capitalistes néolibéraux de la dite postmodernité. Les mots, expression du sujet au moment du danger, sont liés aux sujets concrets de la lutte de classes et aux recompositions des blocs économiques au niveau mondial. Ils ne signifient pas seulement l'insistance d'oublier les espoirs et les morts du passé, ils signifient aussi le désir de liquider les conquêtes de la lutte de classes, de ce qui survit au milieu des réformes structurelles de l'accumulation du capital y compris la mémoire contenant les conquêtes de 1968: une constellation de droits, syndicaux, salariaux, du travail, des retraites et autres formes de redistribution sociale du revenu, etc. Il ne s'agit donc pas d'élucubrations d'un président qui aurait perdu le sens de la négociation institutionnelle.

7 Nous soulignons que ce n'est pas le seul problème de dirigeants et intellectuels européens partageant l'idéologie de l'historicisme insurmontable des vainqueurs annonçant la fin de l'histoire et celle des grands récits depuis 1989. Au Mexique, aussi, existe ce souci de vouloir enterrer la mémoire de l'histoire des vaincus au plus profond de l'oubli. Ricardo Martínez Martínez (2004) mentionne, concernant 1968, que de nombreux acteurs institutionnels insistent sur les logiques de l'histoire du c'est ainsi et relèguent au passé les faits sociaux vidés de leur sens, de la lutte de classes, que 1968 « c'est du passé, c'est fini». Pour l'ex-président Vicente Fox, nous devons oublier le sang versé par les 
stratégies du pouvoir, que "nous allions de l'avant ", et non pas en arrière, ce qui signifie mettre en marche la supposée impartialité de la justice qui, comme nous l'avons vu et vécu au cours de ces dernières années, organise la violence institutionnelle légale et juridique par la militarisation du présent ${ }^{3}$.

Les discours institutionnels et politiques veulent nous assurer de leurs vérités, seuls chemins de leur civilisation. Ils veulent nous convaincre que, dans ce qu'il «reste d'humain » au capitalisme (le travail, la valeur concrète, la valeur d'usage) se trouve la réconciliation permettant d'en terminer avec tant de violence. Ils nous inondent de discours médiatiques sur les faits et les actes violents de cette période, annoncent et insistent que l'injustice est du ressort de certains personnages sans scrupules (exprésidents et militaires sans contrôle - certains encore en vie, d'autres décédés -, de paramilitaires liés à certaines tendances, mais pas au système), qui violèrent la loi et la justice du « système humaniste » du capitalisme et ses justes lois de la guerre.

9 Cependant, en dépit de l'oubli, de sa persistance, des millions de personnes et des collectifs remémorent l'histoire et ses événements. Telle une étincelle, ils allument la vie, attisant l'espoir dissimulé sous les voiles de l'ignominie et du pardon ${ }^{4}$. Si nous nous souvenons de 1968 dans la constellation déterminant les discours du présent, nous pouvons centrer la logique d'oubli et de pardon sur les autorités et les institutions appelant à la réconciliation des contradictions, une synthèse de l'histoire qui avancerait linéairement dans sa modernité pour ne pas revenir sur les significations du passé. Dans ce sens, la date de 1968 et les évènements de l'instant-vécu-espoir donnant vie aux images dialectiques du passé au présent, désirs et aspirations au changement, sont une guerre contre l'oubli, contre la liquidation. Il s'agit d'un oubli inscrit dans la commémoration officielle de l'oubli, un oubli affrontant une constante remémoration du souvenir qui est vécue dans nos corps souffrant les conséquences du totalitarisme devenu démocratie au moyen de discours militarisés.

\section{L'ange de l'histoire en 1968}

Dans l'actualité, se profilent à nouveau les actes funèbres de cette année de douleur et de souvenir. Silence! Des lèvres balbutient les cris des victimes et des regards brillent du feu messianique mouronnant dans les cercueils du passé. Ces expressions, illuminées et profanes, se reflètent dans notre miroir, image de l'éloignement de l'obscurité des lois de la modernité capitaliste. Dans la constellation des luttes des années cinquante des cheminots, enseignants, médecins et étudiants, nous entendons au Mexique les cris du présent proche de la mémoire des victimes de 1968, « le 2 octobre ne s'oublie pas!». Ce sont des sujets de l'histoire, des luttes et des espoirs. En face, d'autres discours se font aussi entendre, ceux de la guerre et de la pax, orchestrés par le pouvoir du Capital présentant ses acteurs : démocratie, justice et liberté.

11 Ainsi, à Mexico, les Commissions et ministères gouvernementaux ${ }^{5}$ parlent de 68 , des décombres qu'il faut déblayer car impossibles à cacher et à évacuer. Le passé de 1968 se présente comme faisant partie du mythe du progrès, impérieux progrès de l'accumulation des temps homogènes vidés des contenus sociaux qui l'ont fait naître. Dans les médias et dossiers de l'histoire, on entend à nouveau que les «mauvais " gouvernants sont ceux qui empêchèrent le triomphe de la démocratie, de la justice et de la liberté. A l'instar des étudiants extrémistes, bien identifiés et classés selon les normes des lois en vigueur, ces diaboliques gouvernants devinrent les ennemis freinant la Modernité. 

humains, des erreurs non calculées par la machinerie triomphante de la civilisation capitaliste. Il s'agit d'injustice systémique, d'erreurs de logique de la marchandise qui doivent être corrigées. Les morts sont la responsabilité de quelques déséquilibrés par le pouvoir et l'ambition de la corruption ${ }^{6}$.

13 L'ex-dirigeant du mouvement étudiant, Jésus Martin del Campo (cité par Saliderna, 2004), affirme que 36 ans après le massacre du 2 octobre, « sonne l'heure » de l'application de la justice et que les responsables doivent être emprisonnés. Comme si le système d'injustice au Mexique pouvait être réformé en profondeur, Martin del Campo et beaucoup d'autres continuent à penser et rêver qu'il est possible d'appliquer la justice dans l'injuste système capitaliste, sui generis. Qui faut-il juger? Les présidents? Les militaires et la police, employés criminels du système? Les juges, ceux qui n'ont jamais rendu justice durant des dizaines ou des centaines d'années et qui ne se posent aucune question sur le système de justice capitaliste? Les avocats, les enseignants et les étudiants de sociologie qui vivent en estimant et en réalisant des valorisations systémiques de l'injustice et de la justice, qui se taisent par ignorance ou par peur des conséquences de la commodité capitaliste postmoderne: bourses, financements privés de fondations, etcetera? Les citoyens complices du passé et du présent gardant le silence face à l'ignominie? En tout cas, et nous insistons, n'est- ce- pas le capitalisme et ses systèmes démocratiques de répartition de justice le responsable de tant de morts tombés pour l'espoir qui les faisait vivre? 1992), les plaies historiques de la lutte de classes ne guérissent pas. Elles restent ouvertes pour interroger le pourquoi de tant de misère, de tant d'injustice et de tant de douleur humaine. Elles ne cicatrisent pas, elles sont les profondes empreintes du Mexique profond. Synonyme de rude, de sauvage, de barbare, de grossier et obstiné, ce Mexique continue à rêver d'une autre vie, d'un Autre Monde et d'un autre temps. Pendant ce temps, s'y accumulent les douleurs, celles des morts d'espoir. Epouvanté par la nature en ruines et par l'animal-homme puis qu'il est nature, l'ange contemple les murs et les pierres de la tragique histoire de Tlatelolco de Cuauhtémoc à 1968. Epouvanté et peiné, il rêve du temps des espoirs du passé dans le présent des vaincus. Il lutte contre l'ouragan du progrès capitaliste ne provoquant que désolations. Il regarde les éclairs de l'histoire et voudrait s'arrêter aux jeux olympiques. Il voudrait miser sur les instants éblouissants de l'histoire se renouvelant dans les expériences des événements du présent.

Ainsi, à la lumière des phares de l'histoire de la résistance, les néo-zapatistes révolutionnaires, héritiers du passé de résistance et de rébellion de la décade des années 60 et de 1968, persistent dans la démesure de vouloir détruire la "bonne politique » autorisée par les institutions. Ce sont des fous, hors du cocktail du stalinisme et sans liens avec ceux qui retournèrent leurs vestes en pleine révolution. Ce jeunes irrévérents vieillissent-juvénilement en croyant que, malgré tout, un autre monde est possible. D'une manière irrationnelle, ils rompent les normes et les règles de la politique, et marchent en bas et à gauche. C'est l'Autre Campagne des zapatistes (Matamoros, 2006) ${ }^{7}$. Parfois, ils se perdent et se trompent mais, c'est avec la patience des révolutionnaires qu'ils attendent dans l'obscurité que les éblouissent les éclairs des orages des nuits du passé. Leurs regards veulent racheter les instants du possible. Ils remémorent les morts pour ne pas mourir dans l'oubli, concentrent les images-éclaires du passé et rassemblent les éclats du miroir brisé de l'histoire pour affronter les mots et les mythes de la démocratie capitaliste.

Variations, 11 | 2008 


\section{Les morts, cheminer avec l'espoir}

16 Dans le contexte de ce que le sous-commandant Marcos appelle la quatrième guerre mondiale, l'humanité s'affronte au néolibéralisme. Les révolutionnaires misent à nouveau pour gagner, mais comme nous l'avons mentionné, ils savent que s'il perdent ni leurs morts ni leur mémoire ne seront en paix. Ainsi, si nous regardons l'année 1968 au Mexique avec Walter Benjamin ne le faisons pas romantiquement dans la mélancolie et les pleurs en croyant que les institutions n'ont pas rempli leur devoir avec l'histoire ou en pensant qu'elles trahissent nos conquêtes. Encore une fois, l'expérience et la connaissance de 1968 doivent être comme les éclairs d'un ciel d'orage car dans l'ombre, leur lumière dessine l'horizon. C'est une luminosité dont on ne peut s'approprier, elle nous permet d'interroger ce totalitarisme dans lequel nous vivons, nuit des erreurs des vaincus dont nous souffrons encore.

Le regard fugace et douloureux de l'ange posé sur le passé nous permet d'interroger ceux qui continuent à croire au progrès et à la justice corporative (traversée par le pouvoir), supposée soigner les blessures et l'ignominie qui se construit sur les institutions juridiques du capitalisme. Il ne faut pas continuer à regarder le passé pour fermer les cicatrices imposées par l'injustice et ses lois promettant justice de génération en génération. De fait, chaque fois que se mentionne ce mot, apparaissent des fantômes, les fantômes lucifériens de nouvelles sanctions de la logique capitaliste génocidaire. Comme Benjamin, il faut brosser l'histoire à rebrousse poils (thèse VII). Il ne s'agit pas de voir les origines de l'injustice ou de la justice mais de regarder avec attention les cicatrices et la profondeur des blessures de l'histoire. Cela permettrait de mettre en relief les antagonismes de l'histoire du pouvoir du capital, de rompre le miroir des identités du pouvoir et de la domination, de voir que la guerre de basse intensité, silence ou moyens des gouvernants, exprime nos forces. L'ange de Walter Benjamin regarde le passé pour éviter la trahison, les erreurs, la récupération du système. Mais, il veut aussi s'arrêter car dans les vents d'en bas contre les vents du progrès existent des espoirs, un rendez-vous, celui de l'histoire du passé avec les nouveaux mouvements sociaux.

La métaphore de l'ange renouvelle les espoirs des instants du temps de l'insurrection révolutionnaire. Se sont les regards des jeunes insurgés en France au cours des dernières années. Se sont les résistances indigènes, accumulées au cours de 500 ans de révoltes, explosant en 1994. Se sont les réseaux de la Commune d'Oaxaca poursuivant leur lutte contre les politiques de militarisations. Se sont les actions qui n'attendent pas que les grandes masses arrivent sur la scène pour changer le monde. Avec les morts du passé et du présent, ces particules sont l'empreinte remémorant les instants de rêves révolutionnaires pour ne pas mourir dans l'oubli réactualisé dans le commémoration des vainqueurs. Ils construisent le temps de la révolte quotidienne contre le monde de l'empire du fétiche de la marchandise. Ils savent, dans leurs tanières et la clandestinité, qu'à chaque instant existe la lumière, éclair du passé projetant les possibles. Dans la clandestinité, imposée par les traités économiques internationaux et les plans de contreinsurrection (voir la militarisation annoncée dans le plan Puebla Panama, actualisé avec le plan Mérida au Mexique repris du plan Colombia), se forme de fissures où s'infiltre l'espoir accumulé dans une histoire pleine de significations. Il ne s'agit pas seulement de résistance, de repousser les vents de la répression, mais d'ouvrir les brèches permettant l'arrivée du Messie (thèse, XVIII, appendice B) pour la rédemption du passé. 

acteurs, dans divers espaces, édition, arts plastiques, poètes du monde, partis, syndicats, communautés indigènes, sortent de leurs tranchées, de leurs jungles contrôlées par la guerre de basse intensité. Ils agissent pour arrêter ce train de l'histoire de douleur, la catastrophe humaine. Contrairement à la limitation du temps de l'histoire officielle, le temps de 1968-2008 n'est ni vide ni homogène. C'est la constellation de la lutte de classes se renouvelant dans un présent plein de luttes de vie contre la violence des desseins de la mort imposée par le capitalisme. Ces dates, années de rébellion, sont les doux rêves de la résistance de l'utopie inscrite dans les cauchemars de l'histoire officielle: condamnation de la guerre dans sa paix- totalitaire du policier et militaire. Elles sont les terribles décomptes des idéologies des comptes configurant les corps robotisés de leur humanité. Mais, elles sont aussi les souvenirs du souvenir du passé dans le présent, vivant les espoirs dans la solitude du silence de la communication monopolisée par les médias mythifiées du totalitarisme de la liberté et de la démocratie institutionnelle et électorale.

Passé d'espoirs et d'utopie du possible des barricades des communes de la liberté de 1968 en France, au Mexique, en Tchécoslovaquie, etcetera, ce temps se renouvelle dans les instants de luttes, à Seattle, Gènes, Porto Allègre et ailleurs, dans les jungles au Chiapas et sur les barricades à Oaxaca et à Atenco. Héritiers de 68, ils se souviennent et, pour ne pas mourir d'insomnie, ils rêvent un autre monde. Comme l'affirme Daniel Bensaï (1990 : 248), remémorant Walter Benjamin, "ne rien oublier nous condamnerait à mourir d'insomnie. Tout oublier nous condamnerait à la servitude sans fin de l'esclave sans mémoire ». Temps messianique, bienvenue maintenant aux plages des rêves des morts du passé, bienvenue à ceux qui croient encore aux significations humaines de la liberté. Bienheureux ceux qui veulent se renouveler dans les significations de la mémoire invisible des arts de la résistance, penser ce qui est pensé pour renouveler l'idée du pas encore, croire et concrétiser qu'il est possible d'arrêter la catastrophe de leur liberté totalitaire.

\section{BIBLIOGRAPHIE}

Aguilar, Manuel, (1989), Huellas del porvenir, 1968-1988, México, Juan Pablos.

Benjamin, Walter, (2000), "Sur le concept d'histoire”, en CEuvres III, Paris, Gallimard.

Bensaïd, Daniel, (1990), Walter Benjamin. Sentinelle Messianique, París, Plon.

Blázquez, Vasni, (1999), “ Un fulgor llamado 68”, en Razón y palabra, núm. 12, octubre.

Bloch, Ernst, (1976), Le Principe Espérance, 3 vols., Paris, Gallimard.

Castillo García, Gustavo, (2004), "Halcones actuaron como francotiradores contra el Ejército el 2 de octubre de 1968 " en La Jornada, México, 2 de octubre

Castillo García, Gustavo, (2004), "No ha habido ni verdad ni justicia en cuanto al 2 de octubre: Guevara Niebla" en La Jornada, México, 2 de octubre.

Debord, Guy, (1992), La société du spectacle, París, Gallimard. 
Editorial, (2006), “2 de octubre, hoy” en La Jornada, México, 2 de octubre.

Escudero, Roberto, (1978), “1968: Pasado y presente” en Cuadernos políticos, núm. 17, julioseptiembre, México, Era.

García, Susana, (1980), “Aproximaciones a un análisis crítico de las hipótesis sobre el movimiento estudiantil de 1968”, en Cuadernos políticos, núm. 25, julio-septiembre, México, Era.

Gómez Bonilla, Edgar, (2007), “Cronología de movimientos estudiantiles en Puebla y la ciudad de México, 1968”, en Tiempo Universitario, núm. 14, año 10, Puebla, octubre.

Guevara Niebla, Gilberto, (1978), “Antecedentes y desarrollo del movimiento de 1968”, en Cuadernos políticos, núm. 17, julio-septiembre, México, Era.

Krivine, Alain, et Daniel Bensaid, (1988), Mai si!, 1968-1988: Rebelles et repentis, Paris, La Brèche.

Martínez Martínez, Ricardo, (2004), “La memoria contra el olvido” en La Jornada, México, 2 de octubre.

Matamoros Ponce, Fernando, (2006), “L'«autre Campagne» des zapatistes” en Le monde Diplomatique, No. 623, Paris, Febrero.

Méndez Ortiz, Alfredo, (2004), “Genocidio, problema jurídico en México, considera experto europeo" en La Jornada, México, 2 de octubre.

Monsiváis, Carlos, (1978), “1968-1978: Cultura y sociedad en México”, en Cuadernos políticos, núm. 17, julio-septiembre, México, Era.

Ramírez, Oscar Omar, (2005), “Acuérdate del 68” en Gaceta CUC, México, octubre.

Saldierna, Georgina, (2004), “Cárcel a culpables” en La Jornada, México, 2 de octubre.

Volpi, Jorge (1998), La imaginación y el poder, México, Era.

Zermeño, Sergio, (1978), "El movimiento estudiantil de 1968”, en Ismael Colmenares et al. (recop.),Cien años de lucha de clases en México (1976-1976), México, Los Autores,.

"Francia, mayo del 68" en: http://redescolar.ilce.edu.mx/redescolar/act_permanentes/historia/ $\mathrm{html} / \mathrm{mov} 68 /$ francia.htm

\section{NOTES}

1. Benjamin Walter, (2000), "Sur le concept d'histoire ", en Oeuvres III, Gallimard, Paris, pp. 427-443. (Toutes les citations de Walter Benjamin seront référenciées dans le texte par le numéro de thèse. $\mathrm{N}$ de $\mathrm{E}$ )

2. “Mai 68, ce n'est pas qu'un début..." est un écho qui vient de loin. C'est un appel international de différentes organisations et personnalités pour agir au présent avec les morts du passé, pour inciter des révoltes contre le néolibéralisme. Des mobilisations sociales se préparent pour le prochain mois de mai 2008. C'est un rendez-vous de l'histoire pour remémorer les morts, réactualiser les urgences du danger quotidien en quarante ans de vie contre la mort de 1968 ( http :// mai- 68.org /).

3. Guerre de basse intensité dans les zones indigènes du Chiapas depuis 1994. Pacification à Oaxaca par la violence militaire contre l'Assemblée Populaire des Peuples d'Oaxaca (APPO) depuis 2006 ayant fait au moins 25 morts et des centaines de prisonniers politiques. Des condamnations à plus de 60 ans de réclusion aux dirigeants d'Atenco dans l'État de Mexico pour avoir lutté depuis 2002 pour empêcher la construction d'un aéroport sur leurs terres ; la presse a mentionné 2 morts, des douzaines de blessés, des viols et plus de 200 arrestations dans ce conflit. Dans l'état 
de Michoacán (20 avril 2006), intervention des forces de l'ordre dans une grève pour évacuer 500 mineurs de l'entreprise sidérurgique Lazaro Cardenas (Sicartsa) à Las Truchas, s'étant soldée par 2 morts et 41 blessés dont 2 gravement. Au Tabasco, la nature n'est pas l'unique responsable des inondations. Celles-ci ont été délibérément provoquées en vidant les retenues d'eau de plusieurs barrages destinés à la production d'énergie hydroélectrique. En effet, en dépit de la période de pluies et des ouragans annoncés, ces réserves avaient été maintenues au maximum de leur capacité. Le gouvernement ne parle pas de morts, les villes sont devenues de villes fantômes, la population qui a survécu crie : ils sont morts, ils (le Capital et le profit) les ont tués.

4. Dans l'actualité, les familles des survivants et disparus se font l'écho des morts réclamant justice en exigeant la création de commissions institutionnelles de la vérité sur cette année de douleur et d'affrontements. Elles dénoncent les méthodes de la démocratie du terrorisme liquidant les opposants de cette période.

5. Le Ministère Spécial des Mouvements Sociaux et Politiques du Passé (la Fiscalia Especial para Movimientos Sociales y Políticos del Pasado FEMOSPP), service du ministère de la justice et du ministère de l'intérieur a commencé son enquête pour rechercher les responsables du génocide perpétré par les autorités en 1968 et 1971, en écartant la responsabilité des gouvernements des États-Unis d'Amérique et de l'Union Soviétique ( $c f$., Castillo Garcia, Gustavo, 2004). Il faut souligner que, dans la mesure où la loi nationale et internationale ne considère pas ce type d'acte comme génocide, aucun génocide ne sera reconnu, et personne ne sera déclaré responsable. C'est la loi et la morale de l'histoire de la lutte de classes.

6. Au total, comme dans les compétitions des jeux olympiques de 1968 , ce n'est pas le système capitaliste qui est responsable des morts du 2 octobre. Ce sont les erreurs humaines particularisées qu'il faut réprimander.

7. Sexta declaración de la selva lacandona, en http://www.ezln.org/documentos/2005/sexta.es.htm, Juin 2005.

\section{AUTEUR}

\section{FERNANDO MATAMOROS}

Chercheur en sociologie, Université de Puebla-Mexico 